\title{
Cardiovascular Risk During the Menopause
}

\author{
María Jesús Cancelo ${ }^{1}$ and Camil Castelo-Branco, ${ }^{2, *}$ \\ ${ }^{1}$ Hospital Universitario de Guadalajara, Universidad de Alcalá de Henares, ${ }^{2}$ Institut Clínic de Ginecologia, Obstetrícia \\ i Neonatologia. Hospital Clínic de Barcelona. IDIBAPS. Facultat de Medicina. Universitat de Barcelona, Spain
}

\begin{abstract}
Although initial experimental data and results of observational studies suggest that hormone replacement therapy (HRT) is associated with a reduction in the risk of heart disease, the results for events such as stroke or thromboembolism are less clear. Randomized secondary and primary prevention studies have found that HRT is not protective against the risk of coronary heart disease, stroke or progression of atherosclerosis. Therefore, HRT therapy should not be initiated to prevent vascular disease among postmenopausal women. There are numerous explanations for the divergent findings of observational and randomized clinical trials of HRT. For example, study design and differing biological effects of HRT on vascular risk factors.
\end{abstract}

The use of HRT for young postmenopausal women with moderate to severe menopausal vasomotor symptoms appears to be safe. The absolute risk of vascular event associated with HRT is low and varies depending on factors like age or years since the menopause. The presence or absence of cardiovascular risk factors determines the differences in vascular risk linked to HRT.

Further studies should identify the mechanisms involved and determine whether and how different regimens of HRT influence vascular risk as well as the influence of individual patient characteristics.

Keywords: Cardiovascular risk, menopause, hormone replacement therapy.

\section{INTRODUCTION}

Menopause may be associated with vasomotor and genital symptoms as well as changes in bone metabolism [1]. Changes in the lipid profile, obesity, hypertension, glucose intolerance and diabetes mellitus may become relevant as risk factors of cardiovascular disease [2]. Vascular disease represents the most frequent cause of mortality and morbidity in the woman, more than cancer either in the United States as in Europe [3].

The risks related to the post-menopausal period are mainly due to the decreased availability of estrogen, which has indirect protective effects on lipid, and glucose metabolism as well as direct effects on vessel function. They have vasodilator action due to nitric oxide (NO) release, calciumantagonist like action and an antiproliferative effect on smooth muscle cells.

In the past, observational data, suggested that estrogen was cardioprotective and as a result, estrogen hormone replacement therapy (HRT) was routinely prescribed for both primary and secondary prevention of coronary heart disease (CHD). However, the Heart and Estrogen Progestin Replacement Study (HERS-I and -II) [4, 5] did not confirm a protective effect on the heart, and the Women's Health Initiative (WHI) combined estrogen-progestin trial reported an

*Address correspondence to this author at the Institut Clínic de Ginecologia, Obstetrícia i Neonatología, Hospital Clínic, Villarroel 170, 08036 Barcelona, Spain; Email: castelobranco@ub.edu; 23247ccb@comb.es increase in CHD risk [6]. The WHI unopposed estrogen trial did not report an increase in CHD events, although stroke and venous thromboembolism were increased similarly in the combined estrogen-progestin trial [7].

However, recent data of follow-up analysis suggests that younger postmenopausal women taking HRT (unopposed or combined) are not at increased risk for CHD [8], and had a lower coronary-plaque burden and a lower prevalence of subclinical coronary artery calcification [9].

HRT is indicated for menopausal symptoms, but it should not be prescribed for either primary or secondary prevention of CHD. The net benefit of estrogen or combined estrogenprogestin HRT in postmenopausal women is still uncertain. We review the impact of estrogen HRT on cardiovascular risk, including the effects on serum lipid values, blood pressure, body weight and cardiovascular outcomes.

\section{CARDIOVASCULAR EFFECTS OF SEXUAL STEROIDS}

\section{Estrogen}

Estrogens have potential beneficial effects on the cardiovascular system such as improving: the lipid profile, insulin sensitivity and endothelial function [10]. Potential adverse effects of estrogens are: Increased serum triglyceride concentrations with oral estrogens, increased hepatic synthesis of vascular inflammatory markers such as C-reactive protein (CRP), prothrombotic effects including an increase in serum fibrinogen, factor VII, and antithrombin III with oral estro- 
gen (less affected by transdermal estrogens) [11]. However, it is not known how these effects influence the overall risk of CHD.

\section{Progestins}

Synthetic progestins, such as medroxyprogesterone acetate, used in the WHI and HERS trials, may counteract some of the beneficial effects of estrogen on lipids and endothelial function [12]. Drospirenone, a synthetic progestin that have antialdosterone activity, has been used for postmenopausal HRT in combination with 17-beta-estradiol (E2) significantly reducing blood pressure in hypertensive women without inducing significant increases in serum potassium [13]. Natural progesterone does not appear to counteract the effects of estrogen on serum lipids.

\section{PRIMARY PREVENTION}

The use of HRT for prevention of disease, particularly CHD was based on observational studies that demonstrated a protective effect of estrogen on the heart [14]. Angiographic and autopsy studies suggested an antiatherogenic effect of estrogens [15].

The WHI trial suggests that estrogen-progestin HRT and unopposed estrogen HRT are not effective for primary prevention of CHD. Combined continuous estrogen-progestin regimen (conjugated estrogen (CE) $0.625 \mathrm{mg}$ and medroxyprogesterone acetate (MPA) $2.5 \mathrm{mg} /$ day) $v s$. placebo in over 16,000 women of 50-79 years old, was discontinued 3 years early due to an increased risk of breast cancer, stroke, CHD, and venous thromboembolism over an average followup of 5.2 years [3] although there were significant benefits in reduction in risk of fractures and colon cancer.

The WHI unopposed estrogen (CE $0.625 \mathrm{mg}$ ) vs. placebo in nearly 11,000 women with hysterectomy was also discontinued (1 year early), due to an increased risk of stroke, and a calculation which suggested no overall health benefit [4].

\section{Combined Estrogen-Progestin}

In the initial reports from the WHI [6], the hazard ratio (HR) for CHD (nonfatal myocardial infarction or death due to $\mathrm{CHD}$ ) in the cohort taking combined estrogen-progestin was 1.24 . However, recent re-analysis suggests that the increased risk may be confined to older women ${ }^{5}$. Other factors, such as body-mass index (BMI), presence of vasomotor symptoms, additional coronary risk factors (including diabetes, hypertension, family history, and smoking), aspirin or statin use, and CRP levels were not significantly related to CHD risk with HRT.

The absolute risk of any adverse event (breast cancer, CHD, stroke or venous thromboembolism) occurring in an individual was extremely low (19 additional events per year per 10,000 women with HRT vs. placebo) [6].

A multicenter trial (WISDOM), similar in design and patient population to the WHI, enrolled 5692 women, but was discontinued after the publication of the early results from the WHI [16]. After a median follow-up of 12 months, an excess risk of CHD events and venous thromboembolism, but not stroke, was observed in the cohort receiving combined estrogen-progestin compared with placebo.

\section{Unopposed Estrogen}

Unlike the excess of CHD risk observed in the combined estrogen-progestin arm of WHI trial, the unopposed estrogens arm, reported a hazard ratio for CHD of 0.95 [4]. This suggests that the progestin played an important role in the increased CHD risk seen with combined therapy [17].

Advers events of unopposed estrogen included an increase in stroke and venous thromboembolic complications (similar to that seen with combined therapy).

\section{Age and Years since Menopause}

These factors may influence the effects on cardiovascular risk as suggested by data from a primate model [18], observational study [19], secondary analyses from the WHI [20] and a meta-analysis of clinical trials [21].

The WHI population was an older population (mean age 63 years). The older age would be expected to be associated with more subclinical atherosclerosis and advanced atherosclerotic lesions that may be more susceptible to the prothrombotic and proinflammatory effects of estrogen. On the other hand, to start estrogen soon after the menopause may result in cardiovascular benefit because women presumably have normal endothelium without atherosclerotic lesions.

The WHI trial, suggested a non significant reduction in CHD risk in women aged 50 to 59 years in the unopposed estrogen trial [19], and in women who started HRT less than 10 years since the menopause in the combined estrogenprogestin trial [22].

For women with $<10,10$ to 19 , or $\geq 20$ years since the menopause, hazard ratios for CHD were $0.76,1.10$, and 1.28 , respectively, with absolute excess risks of $-6,+4$, and +17 per 10,000 person-years [8].

A possible reduction in total mortality was seen in younger women in WHI trial; HR for ages 50 to 50, 60 to 69, and 70 to 79 years were $0.70,1.05$, and 1.14 , respectively [8].

These data should be interpreted with caution, as they are based in surrogate outcomes although there is a suggestion of a possible protective effect of estrogen in younger women. The authors of WHI trial suggest that these data should not change clinical practice and that HRT should not be prescribed for primary prevention of CHD in younger menopausal women [23].

An ongoing trial, the Kronos Early Estrogen Prevention Study, will examine whether earlier initiation of estrogen therapy is associated with a decreased risk of CHD,

\section{Type of Estrogen}

The type of estrogen may also be important in determining risk. A case-control study suggested a possible lower risk of myocardial infarction and stroke with esterified estrogens when compared to conjugated estrogens [24]. 
Both the WHI [6] and the HERS [4] trials employed conjugated equine estrogen-progestin HRT.

The Estrogen in Prevention of Atherosclerosis trial (EPAT) assessed the effect of unopposed 17-beta-estradiol (1 $\mathrm{mg}$ /day) compared with placebo measuring carotid artery intima-media thickness. This study demonstrated that estradiol therapy was associated with a decreased risk of atherosclerosis [25]. On the other hand, when progestin was added to 17-beta-estradiol no reduction in intima-medial progression was show [26].

\section{Route of Estrogen}

Transdermal estrogen has more favourable effects than oral estrogen on markers for cardiovascular risk [27], theoretically being less thrombogenic.

\section{SECONDARY PREVENTION}

Observational and angiographic studies suggested that women with CHD benefit from HRT in terms of survival and preventing subsequent coronary events [28], however clinical trial data have not confirmed these benefits.

The HERS-I trial was a randomized, blinded, placebocontrolled secondary prevention trial [1]. In this study, 2763 postmenopausal women with a history of CHD were randomly assigned to receive the same regimen used in the WHI ( $0.625 \mathrm{mg}$ of conjugated equine estrogen plus $2.5 \mathrm{mg}$ of medroxyprogesterone acetate daily) or placebo for an average of 4 years. More CHD events occurred in the HRT group during the first year of therapy, with a trend for a reduction in risk in years 4 and 5 .

There was no significant difference between the 2 groups in the incidence of CHD events despite a decrease in serum low density lipoprotein cholesterol (LDL-C) concentrations $(11 \%)$ and increase in serum high density lipoprotein cholesterol (HDL-C) concentrations (10\%) in the hormone cohort.

The HERS-II trial was a continuation of HERS-I in an open follow-up study for 2.7 years [2]. Over the 6.8 years of HERS-I and HERS-II, estrogen-progestin HRT did not reduce the risk of CHD events in women with established CHD (HR 0.99). The lower rates of CHD events in the estrogen-progestin group in years 4 and 5 did not persist in the follow-up years.

\section{EFFECTS ON CARDIOVASCULAR RISK MARKERS}

\section{Blood Pressure}

Hypertension is related to increase BMI, with insulinresistance, sodium retention, increased blood viscosity and estrogen deficiency with increased smooth muscle cell proliferation which determines an increase in systemic vascular resistance [29]. Estrogens have little effect on blood pressure [30]. The WHI combined estrogen-progestin trial noted only a small increase $(1.5 \mathrm{mmHg})$ in systolic pressure compared with placebo [3]; a similar difference between the hormone and placebo groups of $1.1 \mathrm{mmHg}$ was noted in the WHI trial of unopposed estrogen [4].

The Postmenopausal Estrogen/Progestin Interventions trial (PEPI), a 3-year, multi-center, randomized, double- blind, placebo-controlled trial consisted of 5 arms: placebo; conjugated equine estrogen (CEE), $0.625 \mathrm{mg} /$ day; CEE, $0.625 \mathrm{mg}$ /day, taken with cyclic MPA $10 \mathrm{mg}$ /day for 12 days a month; CEE, $0.625 \mathrm{mg} /$ day, plus consecutive MPA, 2.5 $\mathrm{mg} /$ day; and CEE, $0.625 \mathrm{mg} /$ day, plus cyclic micronized progesterone (MP), $200 \mathrm{mg}$ /day for 12 days a month, on 875 healthy post-menopausal women aged 45 to 64 years/ The authors found that estrogens, with or without progestins, did not affect blood pressure [31]. Other risk factors were also assessed.

In a recent clinical trial the combined use of drospirenone with 17 beta-estradiol demonstrated a significant blood pressure-lowering effect in untreated hypertension and additive effects when coadministered with ACE inhibitors, angiotensin II type 1 receptor antagonists and thiazide diuretics. In addition, drospirenone reduces bodyweight and lipoprotein concentrations [32].

\section{Body Weight}

Observational trials suggest that the effect of estrogen on body weight is neutral or slightly beneficial. An osteoporosis prevention trial also reported that postmenopausal women receiving HRT gained less weight than women receiving placebo [33] similarly the PEPI trial found that women treated with estrogen gained significantly less weight than those on placebo [21]. A prospective, nonrandomized study of the Rancho Bernardo cohort of 651 women followed for 15 years found that estrogen therapy had no effect on body weight. Abdominal obesity is associated with an increased risk of diabetes, hypertension, and dyslipidemia compared with gynecoid obesity [34]. The effect of hormonal theraphy on body fat distribution was also neutral or favourable [35].

\section{Lipids}

In healthy postmenopausal women oral estrogen has proven a beneficial effect on serum lipid concentrations such as HDL-C and LDL-C but triglyceride levels increase [36]. Estrogens may also lower lipoprotein (a) [LP (a)] levels [37]. These effects appear to be independent of age, with similar results in elderly women [38].

The effect of oral estradiol is similar to oral conjugated equine estrogens while transdermal estradiol has a minor effect compared with oral estrogens because of minor exposure to the liver since avoid the first-pass effect on the liver [39].

In women with hyperlipidemia estrogens were compared with simvastatin; HRT produced a smaller decrease in total cholesterol and LDL-C, a similar elevation in HDL-C, a reduction in LP (a) without change the simvastatin-treated women and an increase in triglycerides $v s$. a decrease with impastation [40]. Similar results were reported when estrogen was compared with simvastatin [41].

The beneficial effects of estrogens on serum lipid concentrations may be counteracted in part by progestin therapy. MPA and levonorgestrel decrease serum HDL-C [42] but the net effect of oral estrogen plus MPA on serum HDL-C is still beneficial, although not as beneficial as when estrogen is given alone [21]. Addition of a progestin has little effect on the estrogen-induced reduction in serum LDL-C levels. 
The type of progestin appears to be important. MPA lowers serum HDL-C much less than levonorgestrel [43]. Oral micronized progetserone seems to have little or no adverse effect [44].

A meta-analysis evaluated the results comparing continuous and cyclic therapy with $0.625 \mathrm{mg}$ conjugated estrogens and MPA. The effects were equivalent with the 2 regimens [45].

\section{STROKE}

The results of epidemiologic studies of estrogen therapy and primary prevention of stroke are conflicting. Estrogens have been related to a benefit [46], no effect [47] and increased risk [48].

One clinical trial suggests that estrogen therapy may increase stroke risk; in the HERS-I trial, there was a strong trend for an increase in risk for fatal stroke (HR 1.61, 95\% CI: 0.97 - 3.55) [49].

In the Women's Estrogen for Stroke Trial (WEST), a double-blind placebo-controlled trial in 664 postmenopausal women with established cerebrovascular disease, oral estradiol $1 \mathrm{mg}$ /day for an average of 2.8 years, had no effect on recurrent stroke or death (HR 1.0) The risk of fatal stroke was (HR 2.9,) [50].

In the WHI trial, increase in stroke risk was seen with combined estrogen-progestin use compared with placebo (HR 1.31). For ischemic and hemorrhagic stroke the hazard ratio were 1.44 , and 0.82 , respectively [51]. The risk was not affected by the presence of diabetes, smoking, biomarkers of inflammation, presence of hypertension, history of cardiovascular disease, or use of statins or aspirin.

In the trial of unopposed estrogen, stroke risk was significantly increased with conjugated CEE vs. placebo (RR 1.3). The excess risk of stroke appeared to be present in women of all ages, including younger and recently menopausal women [52].

In a meta-analysis of randomized trials (including HERS, WEST, and WHI), oral estrogen therapy (with or without progestin) was associated with an increase in ischemic stroke, but not hemorrhagic stroke or transient ischemic attacks (OR 1.29) [53].

The increased risk of stroke associated with HRT, does not appear to be related to the timing of the initiation. In younger women, with lower stroke risk, the risk of stroke attributable to HRT is modest [54].

\section{PERIPHERAL ARTERIAL DISEASE}

Although a population-based study suggested that estrogen therapy for 1 year or more was associated with a decreased risk of peripheral arterial disease [55], this was not confirmed in the HERS-I trial in which therapy with estrogen and progestin did not significantly reduce the incidence of peripheral arterial events (carotid disease, abdominal aortic aneurysm and lower extremity arterial disease [56]). In the WHI, estrogen and progestin did not affect the risk for peripheral arterial events.

\section{VENOUS THROMBOEMBOLISM (VTE)}

One possible mechanism for the greater VTE risk is an increase in activated protein $\mathrm{C}$ resistance that has been reported with both unopposed estradiol and combined estradiol-progestin therapy [57].

Data from observational studies and a meta-analysis suggest that HRT caused approximately a 2-fold increase in VTE risk [58] particularly in women with the factor V Leiden mutation [59].

The WHI trial data are consistent with previous results [60]. Risk of deep vein thrombosis and pulmonary embolism was increased approximately 2 -fold in the estrogen-progestin group compared with placebo, highest in the first year of therapy, but persisting for the 5 year follow-up. Older age and obesity were associated with additional risk, but this was not significantly altered by smoking, aspirin or statin use. The risk was increased in women with the presence of factor $\mathrm{V}$ Leiden but not other prothrombotic mutations. In a casecontrol study of 235 postmenopausal women with VTE and 554 controls, transdermal estrogen, unlike oral estrogen, did not confer additional risk in women who carried a prothrombotic mutation [61].

Unopposed CEE, also increased risk of VTE approximately 2-fold compared with placebo [62].

The risk may be affected by the type of estrogen, route and dose. Transdermal estrogen, which has little effect on hemostasis, may be associated with a lower VTE risk [63]. Neither MPA nor progesterone appeared to be associated with VTE risk [62].

Low-dose HRT, such as $0.3 \mathrm{mg}$ conjugated estrogen, has fewer effects on coagulation and inflammatory markers than standard dose therapy. Higher doses of estrogen, such as those used in oral contraceptives, may be associated with higher VTE risks when compared with HRT doses [64].

\section{CARDIOVASCULAR MORTALITY}

Data on the impact of estrogen HRT on mortality are conflicting. Observational studies reported a reduction in mortality, while the WHI and HERS trials found no effect. A meta-analysis concluded that overall mortality was reduced for users under age 60 years, but not over 60 years [65].

Follow-up analysis combining both WHI trials suggested a reduction in mortality with HRT in younger postmenopausal women.

\section{CONCLUSIONS}

The use of HRT for young postmenopausal women with moderate to severe menopausal vasomotor symptoms appears to be safe. HRT should not be prescribed to postmenopausal women of any age for primary or secondary prevention of CHD.

\section{REFERENCES}

[1] Nelson HD. Menopause. Lancet 2008; 371: 760-70.

[2] Gast GC, Grobbee DE, Pop VJ, et al. Menopausal complaints are associated with cardiovascular risk factors. Hypertension 2008; 51 : 1492-8. 
[3] Wenger NK, Speroff L, Packard B. Cardiovascular health and disease in women. Engl J Med 1993; 329: 247-56.

[4] Hulley S, Grady D, Bush T, et al.; for the Heart and Estrogen/progestin Replacement Study (HERS) Research Group. Randomized trial of estrogen plus progestin for secondary prevention of coronary heart disease in postmenopausal women. JAMA 1998; 280: 605 .

[5] Grady D, Herrington D, Bittner V, et al. Cardiovascular disease outcomes during 6.8 years of hormone therapy: Heart and Estrogen/progestin Replacement Study follow-up (HERS II). JAMA 2002; 288: 49 .

[6] Rossouw JE, Anderson GL, Prentice RL, et al. Risks and benefits of estrogen plus progestin in healthy postmenopausal women: principal results From the Women's Health Initiative randomized controlled trial. JAMA 2002; 288: 321.

[7] Anderson GL, Limacher M, Assaf AR, et al. Effects of conjugated equine estrogen in postmenopausal women with hysterectomy: the Women's Health Initiative randomized controlled trial. JAMA 2004; 291: 1701.

[8] Rossouw JE, Prentice RL, Manson JE, et al. Postmenopausal hormone therapy and risk of cardiovascular disease by age and years since menopause. JAMA 2007; 297: 1465.

[9] Manson JE, Allison MA, Rossouw JE, et al. WHI and WHI-CACS Investigators. Estrogen therapy and coronary-artery calcification. N Engl J Med 2007; 356: 2591-602.

[10] Canonico M, Straczek C, Oger E, et al. Postmenopausal hormone therapy and cardiovascular disease: an overview of main findings. Maturitas 2006; 54: 372-9.

[11] Vehkavaara S, Silveira A, Hakala-Ala-Pietilä T, et al. Effects of oral and transdermal estrogen replacement therapy on markers of coagulation, fibrinolysis, inflammation and serum lipids and lipoproteins in postmenopausal women. Thromb Haemost 2001; 85: 619-25.

[12] Wakatsuki A, Okatani Y, Ikenoue N, et al. Effect of medroxyprogesterone acetate on vascular inflammatory markers in postmenopausal women receiving estrogen. Circulation 2002; 105: 1436-9.

[13] White WB. Drospirenone with 7beta-estradiol in the postmenopausal woman with hypertension. Climacteric 2007; 10 (Suppl 1): 25-31.

[14] Grodstein F, Manson JE, Colditz GA, et al. A prospective, observational study of postmenopausal hormone therapy and primary prevention of cardiovascular disease. Ann Intern Med 2000; 133: 93341.

[15] Christian RC, Harrington S, Edwards WD, et al. Estrogen status correlates with the calcium content of coronary atherosclerotic plaques in women. J Clin Endocrinol Metab 2002; 87: 1062.

[16] Vickers MR, MacLennan AH, Lawton B, et al. Main morbidities recorded in the women's international study of long duration oestrogen after menopause (WISDOM): a randomised controlled trial of hormone replacement therapy in postmenopausal women. BMJ 2007; 335: 239 .

[17] Hulley SB, Grady D. The WHI estrogen-alone trial--do things look any better? JAMA 2004; 291: 1769.

[18] Mikkola TS, Clarkson TB. Estrogen replacement therapy, atherosclerosis, and vascular function. Cardiovase Res 2002; 53: 605.

[19] Grodstein F, Manson JE, Stampfer MJ. Hormone therapy and coronary heart disease: the role of time since menopause and age at hormone initiation. J Womens Health (Larchmt) 2006; 15: 35.

[20] Hsia J, Langer RD, Manson JE, et al. Women's Health Initiative Investigators Conjugated equine estrogens and coronary heart disease: the Women's Health Initiative. Arch Intern Med 2006; 166: 357-65.

[21] Salpeter SR, Walsh JM, Greyber E, et al. Brief report: coronary heart disease events associated with hormone therapy in younger and older women. A meta-analysis. J Gen Intern Med 2006; 21: 363.

[22] Manson JE, Hsia J, Johnson KC, et al. Women's Health Initiative Investigators. Estrogen plus progestin and the risk of coronary heart disease. N Engl J Med 2003; 349: 523-34.

[23] Manson JE, Bassuk SS. Invited commentary: hormone therapy and risk of coronary heart disease why renew the focus on the early years of menopause? Am J Epidemiol 2007; 166: 511.

[24] Lemaitre RN, Weiss NS, Smith NL, et al. Esterified estrogen and conjugated equine estrogen and the risk of incident myocardial infarction and stroke. Arch Intern Med 2006; 166: 399.
[25] Hodis HN, Mack WJ, Lobo RA, et al. Estrogen in the prevention of atherosclerosis: a randomized, double-blind, placebo-controlled trial. Ann Intern Med 2001; 135: 939.

[26] Angerer P, Stork S, Kothny W, et al. Effect of oral postmenopausal hormone replacement on progression of atherosclerosis: a randomized, controlled trial. Arterioscler Thromb Vasc Biol 2001; 21: 262.

[27] Scarabin PY, Alhenc-Gelas M, Plu-Bureau G, et al. Effects of oral and transdermal estrogen/progesterone regimens on blood coagulation and fibrinolysis in postmenopausal women: a randomized controlled trial. Arterioscler Thromb Vasc Biol 1997; 17: 3071.

[28] Shlipak MG, Angeja BG, Go AS, et al. Hormone therapy and inhospital survival after myocardial infarction in postmenopausal women. Circulation 2001; 104: 2300.

[29] Cohen JD. Overview of physiology, vascular biology, and mechanisms of hypertension. J Manag Care Pharm 2007; 13(5 Suppl): S6-8.

[30] Izumi Y, Matsumoto K, Ozawa $\mathrm{Y}$, et al. Effect of age at menopause on blood pressure in postmenopausal women. Am J Hypertens 2007; 20: 1045-50.

[31] Effects of estrogen or estrogen/progestin regimens on heart disease risk factors in postmenopausal women. The Postmenopausal Estrogen/Progestin Interventions (PEPI) Trial. The Writing Group for the PEPI Trial. JAMA 1995; 273: 199-208.

[32] Mallareddy M, Hanes V, White WB. Drospirenone, a new progestogen, for postmenopausal women with hypertension. Drugs Aging 2007; 24: 453-66.

[33] Jensen LB, Vestergaard P, Hermann AP, et al. Hormone replacement therapy dissociates fat mass and bone mass, and tends to reduce weight gain in early postmenopausal women: a randomized controlled 5-year clinical trial of the Danish Osteoporosis Prevention Study. J Bone Miner Res 2003; 18: 333.

[34] Phillips LK, Prins JB. The link between abdominal obesity and the metabolic syndrome. Curr Hypertens Rep 2008; 10: 156-64.

[35] Kritz-Silverstein D, Barrett-Connor E. Long-term postmenopausal hormone use, obesity, and fat distribution in older women. JAMA 1996; $275: 46$.

[36] Walsh BW, Schiff I, Rosner B, et al. Effects of postmenopausal estrogen replacement on the concentrations and metabolism of plasma lipoproteins. N Engl J Med 1991; 325: 1196.

[37] Tuck CH, Holieran S, Berglund L. Hormonal regulation of lipoprotein (a) levels; effects of estrogen replacement therapy on lipoprotein (a) and acute phase reactants in post menopausal women. Arterioscler Thromb Vasc Biol 1997; 17: 1822.

[38] Binder EF, Williams DB, Schechtman KB, et al. Effects of hormone replacement therapy on serum lipids in elderly women: a randomized, placebo-controlled trial. Ann Intern Med 2001; 134: 754.

[39] Walsh BW, Schiff I, Rosner B, et al. Effects of postmenopausal estrogen replacement on the concentrations and metabolism of plasma lipoproteins. Engl J Med 1991; 325: 1196-204.

[40] Darling GM, Johns JA, McCloud PI, et al. Estrogen and progestin compared with simvastatin for hypercholesterolemia in postmenopausal women. N Engl J Med 1997; 337: 595.

[41] Davidson MH, Testolin Lisa, Maki KC, et al. A comparison of estrogen replacement, pravastatin, and combined treatment for the management of hypercholesterolemia in postmenopausal women. Arch Intern Med 1997; 157: 1186

[42] Ottosson UB, Johansson BG, von Schoultz B. Subfractions of highdensity lipoprotein cholesterol during estrogen replacement therapy: a comparison between progestogens and natural progesterone. Am J Obstet Gynecol 1985; 151: 746.

[43] Miller VT, Muesing RA, LaRosa JC, Stoy DB, Phillips EA, Stillman RJ. Obstet Gynecol. Effects of conjugated equine estrogen with and without three different progestogens on lipoproteins, highdensity lipoprotein subfractions, and apolipoprotein A-I. 1991; 77: 235-40.

[44] Moorjani S, Dupont A, Labrie F, et al. Changes in plasma lipoprotein and apolipoprotein composition in relation to oral vs percutaneous administration of estrogen alone or in cyclic association with ultrogestan in menopausal women. J Clin Endocrinol Metab 1991; 73: 373 .

[45] Godsland IF. Effects of postmenopausal hormone replacement therapy on lipid, lipoprotein, and apolipoprotein (a) concentrations: analysis of studies published from 1974-2000. Fertil Steril 2001; 75: 898 . 
[46] Finucane FF, Madans JH, Bush TL, et al. Decreased risk of stroke among postmenopausal hormone users: results from a national cohort. Arch Intern Med 1993; 153: 73.

[47] Angeja BG, Shlipak MG, Go AS, et al. Hormone therapy and the risk of stroke after acute myocardial infarction in postmenopausal women. J Am Coll Cardiol 2001; 38: 1297.

[48] Grodstein F, Manson JE, Colditz GA, et al. A prospective, observational study of postmenopausal hormone therapy and primary prevention of cardiovascular disease. Ann Intern Med 2000; 133: 933.

[49] Simon JA, Hsia J, Cauley JA, et al. Postmenopausal hormone therapy and risk of stroke: the heart and estrogen-progestin replacement study (HERS). Circulation 2001; 103: 638.

[50] Viscoli CM, Brass LM, Kernan WN, et al. A clinical trial of estrogen-replacement therapy after ischemic stroke. N Engl J Med 2001; 345: 1243 .

[51] Wassertheil-Smoller S, Hendrix SL, Limacher M, et al. Effect of estrogen plus progestin on stroke in postmenopausal women: the Women's Health Initiative: a randomized trial. JAMA 2003; 289: 2673.

[52] Hendrix SL, Wassertheil-Smoller S, Johnson KC, et al. Effects of conjugated equine estrogen on stroke in the Women's Health Initiative. Circulation 2006; 113: 2425.

[53] Bath PM, Gray LJ. Association between hormone replacement therapy and subsequent stroke: a meta-analysis. BMJ 2005; 330: 342.

[54] Grodstein F, Manson JE, Stampfer MJ, et al. Postmenopausal hormone therapy and stroke: role of time since menopause and age at initiation of hormone therapy. Arch Intern Med 2008; 168: 861-6.

[55] Westendorp IC, in't Veld BA, Grobbee DE, et al. Hormone replacement therapy and peripheral arterial disease: the Rotterdam study. Arch Intern Med 2000; 160: 2498

[56] Hsia J, Simon JA, Lin F, et al. Peripheral arterial disease in randomized trial of estrogen with progestin in women with coronary heart disease: the Heart and Estrogen/Progestin replacement study. Circulation 2000; 102: 2228.

[57] Post MS, Rosing J, Van Der Mooren MJ, et al. Increased resistance to activated protein $\mathrm{C}$ after short-term oral hormone replacement therapy in healthy post-menopausal women. Br J Haematol 2002; 119: 1017.

[58] Miller J, Chan BK, Nelson HD. Postmenopausal estrogen replacement and risk for venous thromboembolism: a systematic review and meta-analysis for the U.S. Preventive services task force. Ann Intern Med 2002; 136: 680.

[59] Rosendaal FR, Vessey M, Rumley A, et al. Hormonal replacement therapy, prothrombotic mutations and the risk of venous thrombosis. Br J Haematol 2002; 116: 851.

[60] Cushman M, Kuller LH, Prentice R, et al. Estrogen plus progestin and risk of venous thrombosis. JAMA 2004; 292: 1573

[61] Straczek C, Oger E, Beau Yon de Jonage-Canonico M, et al. Prothrombotic mutations, hormone therapy, and venous thromboembolism among postmenopausal women: impact of the route of estrogen administration. Circulation 2005; 112: 3495.

[62] Curb JD, Prentice RL, Bray PF, et al. Venous thrombosis and conjugated equine estrogen in women without a uterus. Arch Intern Med 2006; 166: 772 .

[63] Canonico M, Oger E, Plu-Bureau G, et al. Hormone therapy and venous thromboembolism among postmenopausal women: impact of the route of estrogen administration and progestogens: the ESTHER study. Circulation 2007; 115: 840

[64] Koh KK, Shin MS, Sakuma I, et al. Effects of conventional or lower doses of hormone replacement therapy in postmenopausal women. Arterioscler Thromb Vasc Biol 2004; 24: 1516.

[65] Magliano DJ, Rogers SL, Abramson MJ, et al. Hormone therapy and cardiovascular disease: a systematic review and meta-analysis BJOG 2006; 113: 5-14.

(C) Cancelo and Castelo-Branco; Licensee Bentham Open.

This is an open access article licensed under the terms of the Creative Commons Attribution Non-Commercial License (http://creativecommons.org/licenses/by-nc/3.0/) which permits unrestricted, non-commercial use, distribution and reproduction in any medium, provided the work is properly cited. 